\title{
Study on Elastic Dynamic Model for the Clamping Mechanism of High-Speed Precision Injection Molding Machine
}

\author{
Xue-feng Chen, Jian-guo Hu, Yan-sheng Xu, Zhong-ming Xu, and Hong-bo Wang \\ Department of Mechanical \& Electrical Engineering, Shunde Polytechnic, Foshan 528333, China \\ Correspondence should be addressed to Jian-guo Hu; jghu-sd@139.com
}

Received 24 September 2014; Revised 18 March 2015; Accepted 19 March 2015

Academic Editor: Toshiaki Natsuki

Copyright (C) 2015 Xue-feng Chen et al. This is an open access article distributed under the Creative Commons Attribution License, which permits unrestricted use, distribution, and reproduction in any medium, provided the original work is properly cited.

\begin{abstract}
This work centered on the double-toggle clamping mechanism with diagonal-five points for the high-speed precise plastic injection machine. Based on Lagrange equations, the differential equations of motion for the beam elements are established, in a rotating coordinate system and an absolute coordinate system, respectively. 43 generalized coordinates and a model matrix for the mechanism are created and some coordinate matrices are derived. By coupling the coordinate transformation and matrix manipulation, a high nonlinear and strong time-variant elastic dynamic model is obtained. Based on the dynamic model, a KinetoElasto Dynamics (KED) analysis and a Kineto-Elasto Static (KES) analysis are carried out, respectively. By comparing and analyzing the simulation results of KED and KES, the regularity of elastic vibration of the clamping mechanism in high-speed clamping process has been revealed.
\end{abstract}

\section{Introduction}

The high-speed precision injection molding machine is an advanced molding equipment, which represents the top technical level in the field of plastic molding. The clamping mechanism is the key unit of the machine, which is used for opening, clamping, and locking the plastic mould, and accordingly affects the product quality directly. Due to the good dynamic characteristics, high system stiffness, and large stroke of movable platens, the double-toggle clamping mechanisms are used widely in high-speed precision injection molding machines. And the researches on doubletoggle clamping mechanisms are getting more and more attention. Aiming at the complicated dynamic characteristics of the double-toggle clamping mechanism, Ren et al. [1] built a digital prototype and studied the rigid-flexible coupling dynamic properties. The finite element method and topological optimization method were applied to the design of the clamping mechanism by Kim and Sun [2,3], in order to improve the stiffness and strength and to optimize the structure dimensions. Quan and Liu [4] proposed an adaptive control method to improve the dynamic properties of the clamping mechanism. Pashkevich et al. [5, 6] used stiffness matrices, including the linear stiffness, angular stiffness, and stiffness coupling, to describe the elasticity of the mechanism. Drab and Yilmaz et al. $[7,8]$ used modal synthesis method, multibody dynamics method, and the finite element software, to study the dynamic response of the mechanism. However, owing to the complexity of the structure and kinematics of the double-toggle clamping mechanisms, the existing studies do not exceed the traditional research method for rigid body, which is based on the theory of rigid body dynamics. At the same time, these researches were mainly focused on structure optimization, virtual simulation, strength analysis, stiffness coupling, and so on, which is difficult to explain the complicated dynamic characteristics of the clamping mechanism in high-speed moving process.

With the increasing of the moving velocity and the inertial force during clamping process, the clamping mechanism in the precise plastic injection machine could cause enormous impact, bringing about elastic deformation and excessive vibration. Obviously, the traditional research method for rigid body is unsuited for the new working conditions. Therefore, this work centered on the double-toggle clamping mechanism for high-speed precision injection molding machine began to study the angle of nonlinear vibration, elastic dynamics, and damping theory and built the mathematical 
model of the clamping mechanism, to provide a theoretical basis for the development of high-speed precision injection molding machines.

\section{The Double-Toggle Clamping Mechanism}

In order to build a dynamic model, which can reflect the dynamic characteristics of the clamping mechanism and can be calculated conveniently, some simplification and hypothesis should be made as follows.

(1) All the materials of the parts in the clamping mechanism are uniform and isotropic.

(2) Compared with the nominal displacement of the rigid mechanism obtained by the rigid body kinematics analysis method, the elastic displacement of the elastic mechanism caused by elastic deformation is very small, having little influence on the nominal motion of the mechanism.

(3) The movable platen, stationary platen, and tail platen are considered as rigid bodies and their deformations can be ignored.

(4) All the toggle rods are considered as elastic bodies and are defined as beam elements.

Figure 1 is the structural schematic plot of the doubletoggle clamping mechanism with diagonal-five points, which is discussed in this work.

\section{Nonlinear Elastic Dynamic Model}

3.1. Modeling Ideas. The finite element model is used in the elastic dynamics analysis of the clamping mechanism. First, the clamping mechanism was divided into a number of discrete finite elements. And then a finite element method for mechanism analysis $[9,10]$ was introduced to build the model.

According to the above hypotheses, the actual displacement can be seen as the superposition of the nominal displacement and the elastic displacement. The nominal displacement can be obtained by the rigid body kinematics analysis method, while the elastic displacement can be calculated by the elastic dynamic analysis method [11].

The vibration performances of the clamping system depend on the structure, inertia, elasticity, and dampness of the system itself. In order to build an elastic dynamic model and to reflect the dynamic characteristics of the clamping mechanism, the dynamic substructure analysis method was adopted during the modeling process. First, the clamping system was divided into substructures and elements. Then, the equations of motion of the substructures and elements were built. Finally, these equations of motion were combined into equations of motion for the whole system.

During the dynamic analysis and optimal design process by using the dynamic substructure analysis method, the dynamic models for each substructure must be established firstly. And then according to the mutual connection conditions between the substructures, these dynamic models of

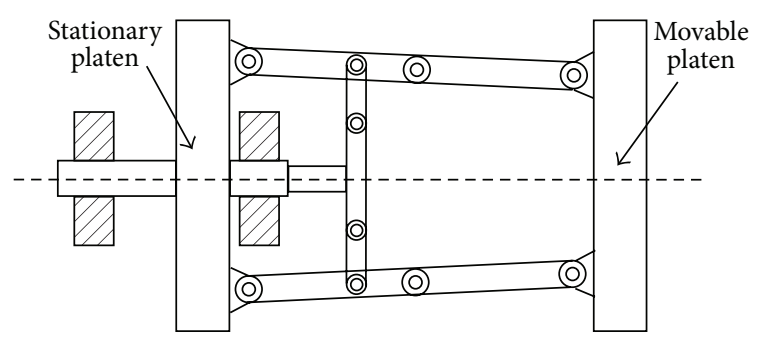

FIGURE 1: The schematic plot of double-toggle clamping unit.

substructures could be integrated into the dynamic model for the whole clamping system.

The detail modeling steps for the double-toggle clamping mechanism with diagonal-five points, shown in Figure 1, are as follows.

(1) According to the shape of the parts and the computational accuracy required, determine the type and number of the elements. For the rod parts of the clamping mechanism, the beam element can be chosen as the element type. By setting nodes at specified points, the double-toggle clamping mechanism can be divided into 13 elements.

(2) Choose nodal displacement mode, create generalized coordinates, and derive the element's differential equations of motion according to Lagrange equations. The elastic displacements and angular displacements at the nodes can be chosen as the generalized coordinates. All the generalized coordinates can be combined as an array, which is the unknown quantity needed to be solved. The number of the generalized coordinates can be described as the DOF of the dynamic model, which determines the scale of the problem solving.

(3) By coordinate transformation, the substructure equations of motion in the physical coordinate can be transformed into ones in the modal coordinate and in uncoupled form. By use of the coordinate conditions of joint points in the joining interface between the substructures, all the mode coordinates of the substructure can be transformed into the coupling mode coordinate of the whole structure. And then the decoupling mathematical model of the whole structure can be obtained by further coordinate transformation. The equation of motion for the clamping system is similar to the one of the vibration system with multiple DOF, as

$$
\mathbf{M U ̈}+\mathbf{C U}+\mathbf{K U}=\mathbf{F}-\mathbf{M} \ddot{\mathbf{U}}_{r},
$$

where $\mathbf{M}, \mathbf{C}$, and $\mathbf{K}$ are the mass matrix, damp matrix, and rigid matrix, respectively. $\mathbf{F}$ is the generalized force matrix. $\ddot{\mathbf{U}}_{r}$ is the acceleration array for rigid body, which can be derived from the rigid motion analysis of the mechanism. $\mathbf{U}, \dot{\mathbf{U}}$, and $\ddot{U}$ are the generalized coordinate array, the according firstorder derivative, and the second-order derivative in time. The generalized coordinate array is the unknown quantity needed to be solved.

3.2. Beam Element. The toggle rods are defined as beam elements and there are two nodes in each beam element. It is assumed that the mass of each element is focused on the axle 


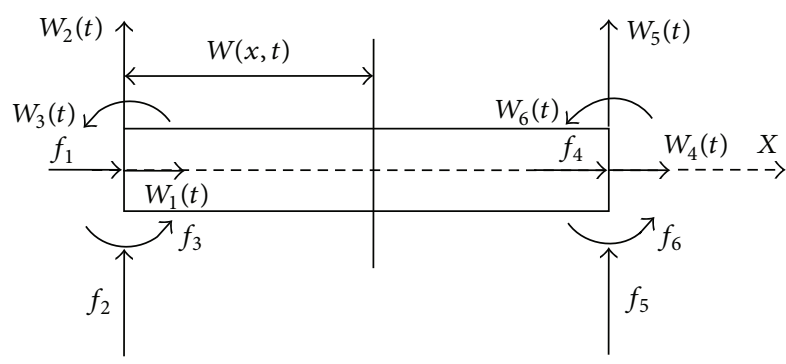

FIgURE 2: The beam element and the nodal displacements.

of the beam and the kinetic energy of the revolving section is ignored. The bending deformation of the beam at the bending moment and the tensile or compressive deformation at the axial load are considered, while the other deformations, such as shearing deformation, are ignored. Therefore, the beam elements are only subjected to the loads from the axial and lateral direction, and there are overall 6 nodal displacements at each endpoint of the beam elements, as shown in Figure 2.

In the figure, $W_{1}(t)$ and $W_{4}(t)$ are nodal longitudinal displacements, $W_{2}(t)$ and $W_{5}(t)$ are nodal lateral displacements, and $W_{3}(t)$ and $W_{6}(t)$ are nodal angular displacements. So, there are 6 generalized coordinates and 6 generalized forces in each beam element.

In order to simplify the solving process, three calculating steps were taken as follows.

First, assume that there are only axial loads applied to the beam element and an axial deformation occurred accordingly. The mass matrix and rigid matrix of this loading condition can be calculated.

Then, assume that there is only lateral loads applied to the beam element and there are only lateral displacements and angular displacements in each node. The mass matrix and rigid matrix can be calculated too.

Finally, superimpose the mass matrices and rigid matrices of the two loading conditions together, respectively, and then the mass matrix and rigid matrix of the beam element subjected to the loads from the axial and lateral direction can be obtained $[12,13]$.

3.3. Equation of Motion of Beam Element. The nodes of the 13 beam elements of the clamping mechanism can be represented by capital letters $\mathrm{A}$ to $\mathrm{N}$ and be identified by numbers (1) to (13). In order to be corresponding to the abovementioned 6 generalized coordinates of the beam element, four other generalized coordinates were set at the joint points of the beam element: two elastic linear displacements along the $X$ and $Y$ direction and two elastic angular displacements. Because the tail platen AE and the hinged supports are considered as rigid bodies, the corresponding two elastic linear displacements are restrained and only the elastic angular displacements are chosen as the generalized coordinates.

For the clamping mechanism discussed in this work, the geometry was meshed with beam elements, as shown in Figure 3. The rod AC is divided as element (1) and element (2) at the joint point $\mathrm{B}$. The rods $\mathrm{CD}$ and $\mathrm{BK}$ are seen as

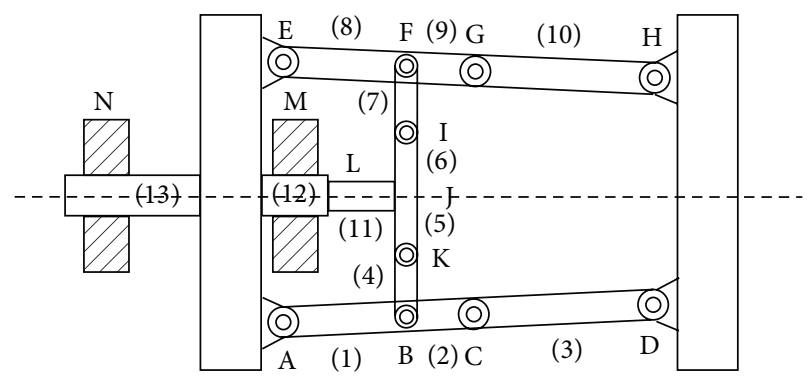

FIGURE 3: Division of clamping mechanism of beam elements.

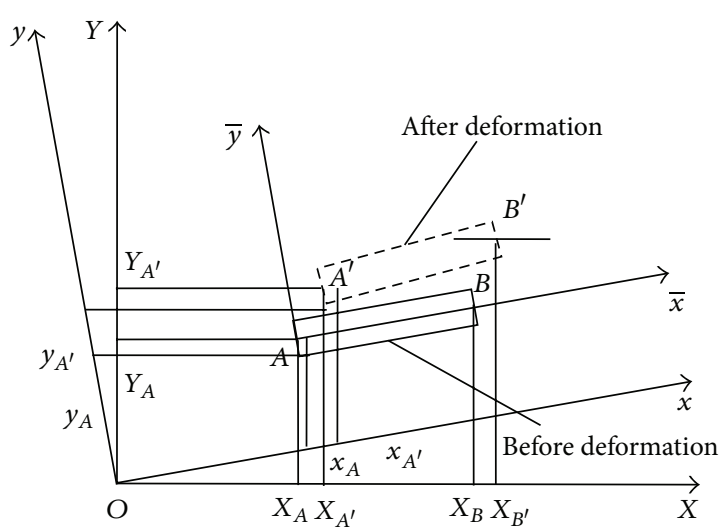

FIGURE 4: The kinematic relations of a beam element before and after deformation.

separate element (3) and element (4), respectively. The rod $\mathrm{KI}$ is divided as element (5) and element (6) at the specified point J. The rod IF is seen as a separate element (7). The rod EG is divided as element (8) and element (9) at the joint point F. The rod GH is seen as a separate element (10). The piston rod $\mathrm{NJ}$ is divided as element (11), element (12), and element (13).

3.3.1. Kinematic Relations of Beam Elements. The instantaneous kinematic analysis method was adopted, which defines the direction and magnitude (translational and rotational) of impending motion of the clamping mechanism at a special point in time. At any special instantaneous time, the mechanism has a special discrete orientation, just like being frozen.

Figure 4 shows the kinematic relations of a beam element before and after deformation. Three coordinate systems are set to analyze its kinematics. The fixed coordinate system $O X Y$ is an absolute coordinate system. The element coordinate system $A \overline{X Y}$ riveted on the beam element is a moving coordinate system. The rotary coordinate system $O x y$ has the same origin as the fixed coordinate system $O X Y$, with coordinate axes parallel to ones of the element coordinate system. At any special instantaneous time, the rotary coordinate system can be seen as a stationary coordinate system.

The element generalized coordinates are the measures in the element coordinate system. In order to facilitate the 
following derivation, the absolute velocity and absolute acceleration in the absolute coordinate system are transformed to the ones in the rotary coordinate system. The transformation relations of velocities are described by

$$
\dot{\mathbf{u}}_{a}=\dot{\mathbf{u}}_{r}+\dot{\mathbf{u}}+\dot{\mathbf{u}}_{r e} .
$$

The vectors in (2), from left to the right, denote the absolute velocity array, the rigid velocity array, the elastic velocity array, and the relative velocity array in the rotary coordinate system, respectively.

Similarly, the transformation relations of accelerations can be described by

$$
\ddot{\mathbf{u}}_{a}=\ddot{\mathbf{u}}_{r}+\ddot{\mathbf{u}}+\ddot{\mathbf{u}}_{n}+\ddot{\mathbf{u}}_{t}+\ddot{\mathbf{u}}_{c} \text {. }
$$

The vectors in (3), from left to the right, denote the absolute acceleration array, the rigid acceleration array, the elastic acceleration array, the relative normal acceleration array, the relative tangential acceleration array, and the Coriolis acceleration array in the rotary coordinate system, respectively.

In the above equations, the vectors $\dot{\mathbf{u}}_{r e}, \ddot{\mathbf{u}}_{n}, \ddot{\mathbf{u}}_{t}$, and $\ddot{\mathbf{u}}_{c}$ are the coupling terms of rigid motion and elastic motion, including both the amount of rigid motion and the amount of elastic motion. In the actual problem-solving situation, however, these coupling terms can be ignored on the conditions of small enough elastic deformation and low enough velocity. Accordingly, (2) and (3) can be briefed as the following equations, respectively,

$$
\begin{aligned}
& \dot{\mathbf{u}}_{a}=\dot{\mathbf{u}}_{r}+\dot{\mathbf{u}} \\
& \ddot{\mathbf{u}}_{a}=\ddot{\mathbf{u}}_{r}+\ddot{\mathbf{u}} .
\end{aligned}
$$

Equations (4) and (5) imply the hypothesis in Section 2, which can be represented as that the actual motion (that is the absolute motion) of the clamping mechanism is the superimposition of the rigid motion and the elastic motion.

\subsubsection{Differential Equations of Motion of Beam Elements} in Different Coordinate Systems. The following equation is Lagrange's equation:

$$
\frac{d}{d t}\left(\frac{\partial E_{k}}{\partial u_{j}}\right)-\frac{\partial E_{k}}{\partial u_{j}}-F_{j}=0 .
$$

When deriving the equation of motion of mechanical system by means of Lagrange's equation, it is not necessary to consider the constraint forces acting on the system. It is advantageous and efficacious in the practical application, especially for the mechanical system with a high number of DOF. All the differential equations are derived from the Lagrangian scalar function and virtual work equation of nonconservative forces. The main steps are as follows: first, determine the number of DOF of the system; then, choose generalized coordinates; finally, express the kinetic energy and potential energy of the system with the generalized coordinates and generalized velocities.

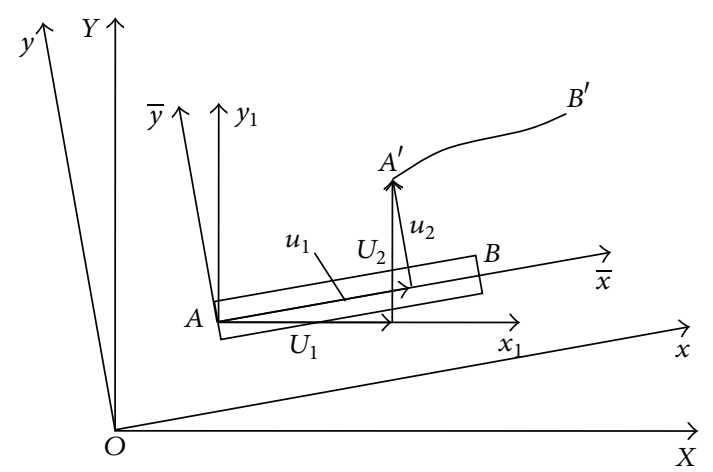

FIGURE 5: Coordinate transformation.

The following is the deriving process of dynamic equations for the clamping mechanism by means of Lagrange's equations.

(1) Differential equations of motion of beam elements in the rotary coordinate system $O x y$ are as follows.

Based on Lagrange's equation, the differential equations of motion of beam elements in rotary coordinate system $O x y$ can be given directly by

$$
\overline{\mathbf{m}} \ddot{\mathbf{u}}+\overline{\mathbf{k}} \mathbf{u}=\overline{\mathbf{f}}-\overline{\mathbf{m}} \ddot{\mathbf{u}}_{r},
$$

where $\mathbf{u}$ is a generalized coordinate defined in the moving coordinate system $A \overline{X Y}$ and $\ddot{\mathbf{u}}_{r}$ is the rigid acceleration array defined in the rotary coordinate system.

(2) Differential equations of motion of beam elements in the absolute coordinate system $O X Y$ are as follows.

In order to assemble the equations of motion of element into the equations of motion of system, it is needed to choose the nodal linear displacements, parallel to the coordinate axes of the absolute coordinate system, as generalized coordinates. Accordingly, a new element generalized coordinate array is introduced as the following array and is shown in Figure 5:

$$
\mathbf{U}^{e}=\left[\begin{array}{llllll}
\mathbf{U}_{1} & \mathbf{U}_{2} & \mathbf{U}_{3} & \mathbf{U}_{4} & \mathbf{U}_{5} & \mathbf{U}_{6}
\end{array}\right] .
$$

Here, it is necessary to illustrate the significance of generalized coordinate array $\mathbf{U}^{e}$. The two coordinate systems, set at the two nodes of the moving beam element, will move along with the rigid beam element, while the coordinate axes of the two coordinate systems will always be parallel to ones of the absolute coordinate system. The two coordinate systems can be called translational coordinate systems. The array elements of $\mathbf{U}^{e}$ are defined in the two translational coordinate systems.

Obviously, the two groups of coordinates in the rotary coordinate system and in the absolute coordinate system, 


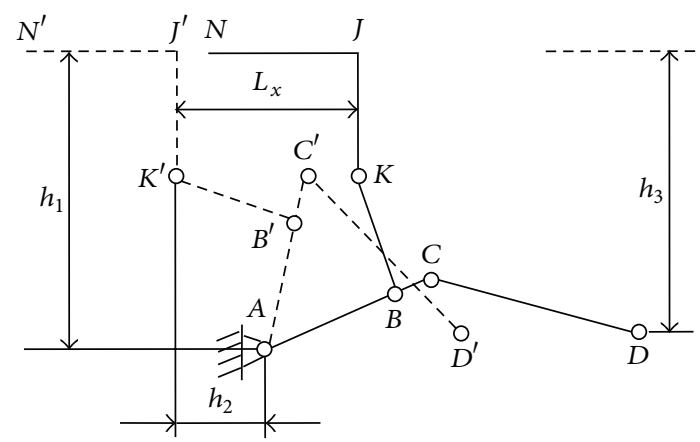

Figure 6: The schematic diagram of the clamping mechanism.

respectively, have the following relationships as in the formula

$$
\begin{aligned}
& u_{1}=U_{1} C+U_{2} S \\
& u_{2}=U_{1} S+U_{2} C \\
& u_{3}=U_{3} \\
& u_{4}=U_{4} C+U_{5} S \\
& u_{5}=U_{4} S+U_{5} C \\
& u_{6}=U_{6},
\end{aligned}
$$

where $S=\sin \theta$ and $C=\cos \theta$. Formula (9) can be written as the following formula in matrix form:

$$
\mathbf{u}=\mathbf{R U}^{e}
$$

In formula (10), $\mathbf{R}$ is the coordinate transformation matrix as formula (11). For different beam element, the coordinate transformation matrix is different:

$$
\mathbf{R}=\left[\begin{array}{cccccc}
C_{i} & S_{i} & 0 & 0 & 0 & 0 \\
-S_{i} & C_{i} & 0 & 0 & 0 & 0 \\
0 & 0 & 1 & 0 & 0 & 0 \\
0 & 0 & 0 & C_{i} & S_{i} & 0 \\
0 & 0 & 0 & -S_{i} & C_{i} & 0 \\
0 & 0 & 0 & 0 & 0 & 1
\end{array}\right] \quad(i=1,2, \ldots 13)
$$

Figure 6 is the schematic diagram of the clamping mechanism. Because the upper part and the lower part of the mechanism are symmetric in structure, only the lower half part is shown in Figure 6. The dotted lines denote the initial position of the clamping mechanism, while the solid lines denote any other position.

The clamping mechanism is divided into 13 beam elements, and accordingly there are 13 coordinate transformation matrices with different horizontal angles $\theta$. The following is the calculating process of the horizontal angles, which can be substituted into formula (11) to calculate the coordinate transformation matrices. Refer to Figures 3 and 6, and ignore the complicated derivation process; the horizontal angle $\theta_{i}$ can be calculated by the following formulas:

$$
\begin{aligned}
\theta_{1}=\operatorname{tg}^{-1}\left(\frac{\left(L_{x}-h_{2}\right)^{2}\left(h_{1}-L_{J K}\right)}{\left(\left(L_{x}-h_{2}\right)^{2}-L_{K B}^{2}\right)\left(L_{x}-h_{2}\right)}\right. \\
+\left(4\left(L_{x}-h_{2}\right)^{4}\left(h_{1}-L_{J K}\right)^{2}\right. \\
\quad-4\left(\left(L_{x}-h_{2}\right)^{2}-L_{K B}^{2}\right)\left(\left(L_{x}-h_{2}\right)^{2}-L_{K B}^{2}\right) \\
\left.\cdot\left(L_{x}-h_{2}\right)^{2}\right)^{1 / 2}
\end{aligned}
$$

$$
\left.\cdot\left(2\left(\left(L_{x}-h_{2}\right)^{2}-L_{K B}^{2}\right)\left(L_{x}-h_{2}\right)\right)^{-1}\right)
$$

$$
\theta_{2}=\theta_{1}
$$

$$
\begin{gathered}
\theta_{3}=180^{\circ}-\sin ^{-1}\left(\frac{L_{A C} \sin \theta_{1}-h_{1}+h_{3}}{L_{C D}}\right) \\
\theta_{4}=180^{\circ}-\sin ^{-1}\left(\frac{h_{1}-L_{J K}-L_{A B} \sin \theta_{1}}{L_{K B}}\right) \\
\theta_{5}=90^{\circ} \\
\theta_{6}=90^{\circ} \\
\theta_{7}=-\sin ^{-1}\left(\frac{h_{1}-L_{J K}-L_{A B} \sin \theta_{1}}{L_{K B}}\right) \\
\begin{array}{c}
\left(L_{x}-h_{2}\right)^{2}\left(h_{1}-L_{J K}\right) \\
\left(\left(L_{x}-h_{2}\right)^{2}-L_{K B}^{2}\right)\left(L_{x}-h_{2}\right) \\
+\left(4\left(L_{x}-h_{2}\right)^{4}\left(h_{1}-L_{J K}\right)^{2}\right. \\
-4\left(\left(L_{x}-h_{2}\right)^{2}-L_{K B}^{2}\right) \\
\left.\cdot\left(\left(h_{1}-L_{J K}\right)^{2}-L_{K B}^{2}\right)\left(L_{x}-h_{2}\right)^{2}\right)^{1 / 2} \\
\cdot\left(2\left(\left(L_{x}-h_{2}\right)^{2}-L_{K B}^{2}\right)\left(L_{x}-h_{2}\right)\right)^{-1} \\
\theta_{9}=\theta_{8} \\
\theta_{10}=\sin ^{-1}\left(\frac{L_{A C} \sin \theta_{1}-h_{1}+h_{3}}{L_{C D}}\right) \\
\theta_{11}=0^{\circ} \\
\theta_{12}=0^{\circ} \\
\theta_{13}=0^{\circ} .
\end{array}
\end{gathered}
$$

For one mechanism, when different sets of generalized coordinate are chosen, different equations of motion will be obtained and different coupling modes will be adopted. The coupling of equations of motion is not the form of the motion 


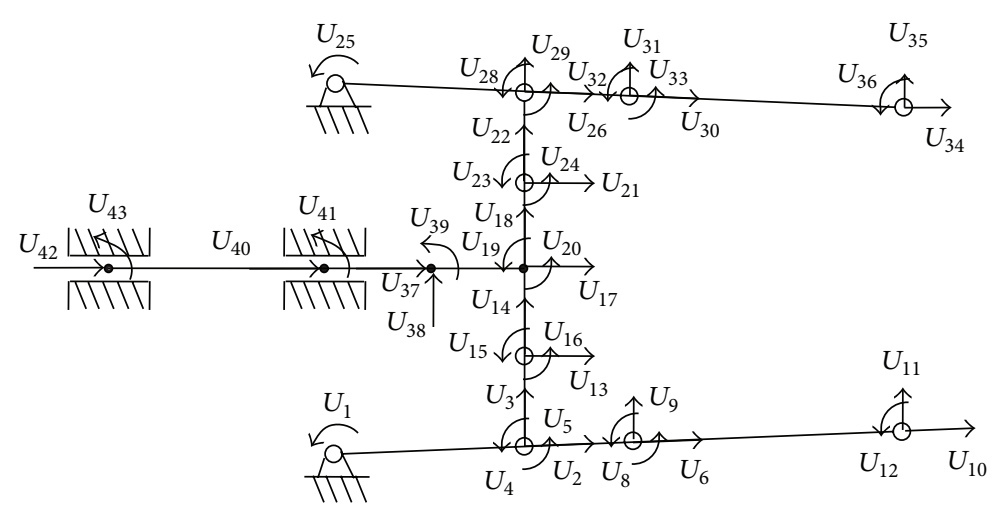

FIgURE 7: The finite element model of the clamping mechanism.

system but the result of the selection of the generalized coordinates. Natural coordinate is a set of generalized coordinate, which will obtain equation of motion in uncoupled form. Any set of generalized coordinate can be transformed into natural coordinate by linear transformation using a modal transformation matrix, which will make the uncoupling of the equations become more convenient.

Define the following array:

$$
\ddot{\mathbf{U}}_{r}^{e}=\left[\begin{array}{llllll}
\ddot{\mathbf{X}}_{\mathbf{A}} & \ddot{\mathbf{Y}}_{\mathbf{A}} & \ddot{\theta} & \ddot{\mathbf{X}}_{\mathbf{B}} & \ddot{\mathbf{Y}}_{\mathbf{B}} & \ddot{\theta}
\end{array}\right]
$$

$\ddot{\mathbf{U}}_{r}^{e}$ is the rigid acceleration array of rigid beam element in the absolute coordinate system. $\ddot{\mathbf{u}}_{r}$ is the rigid acceleration array in rotary coordinate system. They all are fixed coordinate system and have the relationships as

$$
\begin{aligned}
\ddot{\mathbf{u}} & =\mathbf{R} \ddot{\mathbf{U}}^{e} \\
\ddot{\mathbf{u}}_{r} & =\mathbf{R} \ddot{\mathbf{U}}_{r}^{e},
\end{aligned}
$$

where $\mathbf{R}$ is a symmetric square matrix.

By (7), the following equation can be obtained:

$$
\overline{\mathbf{m}} \mathbf{R} \ddot{\mathbf{U}}^{e}+\overline{\mathbf{k}} \mathbf{R U}=\overline{\mathbf{f}}-\overline{\mathbf{m}} \mathbf{R} \ddot{\mathbf{U}}_{r}^{e} .
$$

In order to keep the symmetry of the equation, we can multiply "on the left" on both sides of the equation by $\mathbf{R}^{\mathbf{T}}$. And the following equation can be obtained:

$$
\mathbf{R}^{\mathrm{T}} \overline{\mathbf{m}} \mathbf{R} \ddot{\mathbf{U}}^{e}+\mathbf{R}^{\mathrm{T}} \overline{\mathbf{k}} \mathbf{R} \mathbf{U}=\mathbf{R}^{\mathrm{T}} \overline{\mathbf{f}}-\mathbf{R}^{\mathrm{T}} \overline{\mathbf{m}} \mathbf{R} \ddot{\mathbf{U}}_{r}^{e} .
$$

Equation (16) can be written in a simplified form as

$$
\mathbf{m} \ddot{\mathbf{U}}^{e}+\mathbf{k} \mathbf{U}=\mathbf{f}-\mathbf{m} \ddot{\mathbf{U}}_{r}^{e}
$$

where

$$
\begin{aligned}
\mathbf{m} & =\mathbf{R}^{\mathrm{T}} \overline{\mathbf{m}} \mathbf{R} \\
\mathbf{k} & =\mathbf{R}^{\mathrm{T}} \overline{\mathbf{k}} \mathbf{R} .
\end{aligned}
$$

$\mathbf{m}$ and $\mathbf{k}$ are the mass matrix and rigid matrix in the generalized coordinates, respectively. Due to $\overline{\mathbf{m}}$ and $\overline{\mathbf{k}}$ being symmetric matrices, $\overline{\mathbf{m}}$ and $\overline{\mathbf{k}}$ are symmetric ones. Therefore, we can know that the influence of coordinate transformation on equation of motion is the corresponding transformations of mass matrix and rigid matrix. Consider

$$
\mathbf{f}=\mathbf{R}^{\mathrm{T}} \overline{\mathbf{f}}
$$

Equation (19) is the generalized force matrix along the component direction of generalized coordinate $\mathbf{u}^{e}$. The generalized force matrix includes both the external force and the internal force acting on the element nodes by adjacent elements.

The above matrix transformations can transform the coordinates between different coordinate systems but cannot change the nature of the motion system. The transformations of mass matrix and rigid matrix, however, could change the coupling situation of the equation of motion. Equation (17) is the differential equations of motion of beam elements in the absolute coordinate system.

\subsection{The Dynamic Equation of the System}

3.4.1. The Generalized Coordinates of the System. Based on the generated mesh of beam element, the finite element model for the double-toggle clamping mechanism was established, which includes 43 generalized coordinates, as shown in Figure 7.

The 43 generalized coordinates can be denoted as $U_{1}, U_{2}, U_{3} \cdots U_{42}, U_{43}$. Here, the subscript numbers are system numbers or global numbers. In the element generalized coordinate array $\mathbf{U}^{e}$, the subscript numbers $1,2,3,4,5$, and 6 are element numbers or local numbers. Therefore, the relations between the global numbers and local numbers must be built. It can be realized by an integral matrix $\mathbf{I}_{\mathcal{u}}$, also called model matrix. The row number in the matrix is equal to total number of the element, and the column number is equal to the number of the generalized coordinates.

As for the double-toggle clamping mechanism discussed in this work, the model matrix is a $13 \times 6$ matrix, as shown in formula (20). In the $13 \times 6$ model matrix, the six numbers at the $i$ th row are the corresponding system numbers of the local numbers 1 to 6 in the ith element. The zero value elements in the model matrix indicate that the corresponding generalized 
coordinates are not set. For example, for the third element, the generalized coordinate array can be obtained as formula

$$
\begin{aligned}
& \mathbf{I}_{u}=\left[\begin{array}{cccccc}
0 & 0 & 1 & 2 & 3 & 4 \\
2 & 3 & 4 & 6 & 7 & 8 \\
6 & 7 & 9 & 10 & 11 & 12 \\
2 & 3 & 5 & 13 & 14 & 15 \\
13 & 14 & 16 & 17 & 18 & 19 \\
17 & 18 & 19 & 21 & 22 & 23 \\
21 & 22 & 24 & 26 & 27 & 28 \\
0 & 0 & 25 & 26 & 27 & 28 \\
26 & 27 & 29 & 30 & 31 & 32 \\
30 & 31 & 33 & 34 & 35 & 36 \\
37 & 38 & 39 & 17 & 18 & 20 \\
40 & 0 & 41 & 37 & 38 & 39 \\
42 & 0 & 43 & 40 & 0 & 41
\end{array}\right] \\
& \mathbf{U}_{3}^{e}=\left[\begin{array}{llllll}
\mathbf{U}_{6} & \mathbf{U}_{7} & \mathbf{U}_{9} & \mathbf{U}_{10} & \mathbf{U}_{11} & \mathbf{U}_{12}
\end{array}\right] .
\end{aligned}
$$

3.4.2. The Differential Equation of Motion of the System. The subscript numbers in the above differential equations of motion of beam elements are local numbers. Such equations of motion can be written for each element. Therefore, there are 13 such element equations for the 13 beam elements. By gathering the 13 element equations, the differential equations of motion of the clamping system can be obtained. For the $i$ th beam element, the differential equation of motion can be written as formula

$$
\mathbf{m}_{i} \ddot{\mathbf{U}}_{i}^{e}+\mathbf{k}_{i} \mathbf{U}_{i}^{e}=\mathbf{f}_{i}-\mathbf{m}_{i} \ddot{\mathbf{U}}_{r i}^{e} \quad(i=1,2,3 \cdots 13),
$$

where the subscript $i$ denotes the amount of the $i$ th beam elements.

The generalized coordinate array of the clamping system can be defined as

$$
\mathbf{U}=\left[\begin{array}{lllll}
\mathbf{U}_{1} & \mathbf{U}_{2} & \mathbf{U}_{3} & \cdots & \mathbf{U}_{43}
\end{array}\right]^{\mathrm{T}},
$$

where subscript 43 is the total number of the generalized coordinates, equal to the number of DOF of the clamping system.

The rigid velocity array of the clamping unit can be defined as

$$
\dot{\mathbf{U}}_{r}=\left[\begin{array}{ccccccccc}
\dot{\theta}_{1} & \dot{\mathbf{X}}_{2} & \dot{\mathbf{Y}}_{2} & \dot{\theta}_{1} & \dot{\theta}_{3} & \dot{\mathbf{X}}_{3} & \dot{\mathbf{Y}}_{3} & \dot{\theta}_{1} & \dot{\theta}_{2} \\
\dot{\mathbf{X}}_{4} & \dot{\mathbf{Y}}_{4} & \dot{\theta}_{2} & \dot{\mathbf{X}}_{5} & \dot{\mathbf{Y}}_{5} & \dot{\theta}_{3} & \dot{\theta}_{4} & \dot{\mathbf{X}}_{6} & \dot{\mathbf{Y}}_{6} \\
\dot{\theta}_{4} & \dot{\theta}_{8} & \dot{\mathbf{X}}_{7} & \dot{\mathbf{Y}}_{7} & \dot{\theta}_{4} & \dot{\theta}_{5} & \dot{\theta}_{6} & \dot{\mathbf{X}}_{8} & \dot{\mathbf{Y}}_{8} \\
\dot{\theta}_{5} & \dot{\theta}_{6} & \dot{\mathbf{X}}_{9} & \dot{\mathbf{Y}}_{9} & \dot{\theta}_{6} & \dot{\theta}_{7} & \dot{\mathbf{X}}_{10} & \dot{\mathbf{Y}}_{10} & \dot{\theta}_{7} \\
\dot{\mathbf{X}}_{11} & \dot{\mathbf{Y}}_{11} & \dot{\theta}_{8} & \dot{\mathbf{X}}_{12} & \dot{\theta}_{8} & \dot{\mathbf{X}}_{13} & \dot{\theta}_{8} & &
\end{array}\right]
$$

The arrays $\dot{\mathbf{U}}_{r}$ and $\mathbf{U}$ have the same number of array dimension, and the corresponding elements have the following relations.
If $\mathbf{U}_{i}$ is the elastic linear displacement in $X$ or $Y$ direction, then $\dot{\mathbf{U}}_{r i}$ is the nodal rigid velocity in the same direction. While if $\mathbf{U}_{i}$ is the elastic angular displacement, then $\dot{\mathbf{U}}_{r i}$ is the angular velocity of the part where the element is generated.

The rigid acceleration array of the clamping system can be defined as

$$
\ddot{\mathbf{U}}_{r}=\left[\begin{array}{lllll}
\ddot{\mathbf{U}}_{r 1} & \ddot{\mathbf{U}}_{r 2} & \ddot{\mathbf{U}}_{r 3} & \cdots & \ddot{\mathbf{U}}_{r 43}
\end{array}\right]^{\mathbf{T}} .
$$

Similarly, the arrays $\ddot{\mathbf{U}}_{r}$ and $\mathbf{U}$ have the same number of array dimension, and the corresponding elements have the following relations.

If $\mathbf{U}_{i}$ is the elastic linear displacement in $X$ or $Y$ direction, then $\ddot{\mathbf{U}}_{r i}$ is the nodal rigid acceleration in the same direction. While if $\mathbf{U}_{i}$ is the elastic angular displacement, then $\ddot{\mathbf{U}}_{r i}$ is the angular acceleration of the part where the element is generated.

As for the double-toggle clamping mechanism discussed in this work, the rigid acceleration array can be written as formula

$$
\ddot{\mathbf{U}}_{r}=\left[\begin{array}{ccccccccc}
\ddot{\theta}_{1} & \ddot{\mathbf{X}}_{2} & \ddot{\mathbf{Y}}_{2} & \ddot{\theta}_{1} & \ddot{\theta}_{2} & \ddot{\mathbf{X}}_{3} & \ddot{\mathbf{Y}}_{3} & \ddot{\theta}_{1} & \ddot{\theta}_{3} \\
\ddot{\mathbf{X}}_{4} & \ddot{\mathbf{Y}}_{4} & \ddot{\theta}_{3} & \ddot{\mathbf{X}}_{5} & \ddot{\mathbf{Y}}_{5} & \ddot{\theta}_{2} & \ddot{\theta}_{4} & \ddot{\mathbf{X}}_{6} & \ddot{\mathbf{Y}}_{6} \\
\ddot{\theta}_{4} & \ddot{\theta}_{5} & \ddot{\mathbf{X}}_{7} & \ddot{\mathbf{Y}}_{7} & \ddot{\theta}_{4} & \ddot{\theta}_{6} & \ddot{\theta}_{7} & \ddot{\mathbf{X}}_{8} & \ddot{\mathbf{Y}}_{8} \\
\ddot{\theta}_{6} & \ddot{\theta}_{7} & \ddot{\mathbf{X}}_{9} & \ddot{\mathbf{Y}}_{9} & \ddot{\theta}_{7} & \ddot{\theta}_{8} & \ddot{\mathbf{X}}_{10} & \ddot{\mathbf{Y}}_{10} & \ddot{\theta}_{8} \\
\ddot{\mathbf{X}}_{11} & \ddot{\mathbf{Y}}_{11} & \ddot{\theta}_{5} & \ddot{\mathbf{X}}_{12} & \ddot{\theta}_{5} & \ddot{\mathbf{X}}_{13} & \ddot{\theta}_{5} & &
\end{array}\right]^{2}
$$

$\mathbf{U}_{i}^{e}$ is a 6-dimensional array, and $\mathbf{U}$ is a 43-dimensional array. The former is a part of the latter. The relation between the two arrays can be denoted by formula

$$
\mathbf{U}_{i}^{e}=\mathbf{B}_{i} \mathbf{U},
$$

where $\mathbf{B}_{i}$ is called coordinate matrix and is a $6 \times 43$ matrix in which each element is 0 or 1 . The coordinate matrix can be generated on the base of the model matrix.

For the 13 beam elements of the double-toggle clamping mechanism, 13 coordinate matrices can be derived as formula

$$
\begin{gathered}
\mathbf{B}_{1}=\left[\begin{array}{llllll}
0 & 0 & 0 & 0 & & \\
0 & 0 & 0 & 0 & & \\
1 & 0 & 0 & 0 & & 0 \\
0 & 1 & 0 & 0 & (6 \times 39) \\
0 & 0 & 1 & 0 & & \\
0 & 0 & 0 & 1 & &
\end{array}\right] \\
\mathbf{B}_{2}=\left[\begin{array}{lllllllll}
0 & 1 & 0 & 0 & 0 & 0 & 0 & 0 \\
0 & 0 & 1 & 0 & 0 & 0 & 0 & 0 \\
0 & 0 & 0 & 1 & 0 & 0 & 0 & 0 \\
0 & 0 & 0 & 0 & 0 & 1 & 0 & 0 & 0 \\
0 & 0 & 0 & 0 & 0 & 0 & 1 & 0 \\
0 & 0 & 0 & 0 & 0 & 0 & 0 & 1
\end{array}\right]
\end{gathered}
$$




$$
\begin{aligned}
& \mathbf{B}_{3}=\left[\begin{array}{ccccccccc} 
& 1 & 0 & 0 & 0 & 0 & 0 & 0 & \\
& 0 & 1 & 0 & 0 & 0 & 0 & 0 & \\
0 & 0 & 0 & 0 & 1 & 0 & 0 & 0 & 0 \\
(6 \times 5) & 0 & 0 & 0 & 0 & 1 & 0 & 0 & (6 \times 31) \\
& 0 & 0 & 0 & 0 & 0 & 1 & 0 & \\
& 0 & 0 & 0 & 0 & 0 & 0 & 1
\end{array}\right] \\
& \mathbf{B}_{4}=\left[\begin{array}{cccccccccc}
0 & 1 & 0 & 0 & 0 & & 0 & 0 & 0 & \\
0 & 0 & 1 & 0 & 0 & & 0 & 0 & 0 & \\
0 & 0 & 0 & 0 & 1 & 0 & 0 & 0 & 0 & 0 \\
0 & 0 & 0 & 0 & 0 & (6 \times 7) & 1 & 0 & 0 & (6 \times 28) \\
0 & 0 & 0 & 0 & 0 & & 0 & 1 & 0 & \\
0 & 0 & 0 & 0 & 0 & & 0 & 0 & 1
\end{array}\right] \\
& \mathbf{B}_{5}=\left[\begin{array}{ccccccccc} 
& 1 & 0 & 0 & 0 & 0 & 0 & 0 & \\
& 0 & 1 & 0 & 0 & 0 & 0 & 0 & \\
0 & 0 & 0 & 0 & 1 & 0 & 0 & 0 & 0 \\
(6 \times 12) & 0 & 0 & 0 & 0 & 1 & 0 & 0 & (6 \times 24) \\
& 0 & 0 & 0 & 0 & 0 & 1 & 0 & \\
& 0 & 0 & 0 & 0 & 0 & 0 & 1
\end{array}\right] \\
& \mathbf{B}_{6}=\left[\begin{array}{ccccccccc} 
& 1 & 0 & 0 & 0 & 0 & 0 & 0 & \\
& 0 & 1 & 0 & 0 & 0 & 0 & 0 & \\
0 & 0 & 0 & 1 & 0 & 0 & 0 & 0 & 0 \\
(6 \times 16) & 0 & 0 & 0 & 0 & 1 & 0 & 0 & (6 \times 21) \\
& 0 & 0 & 0 & 0 & 0 & 1 & 0
\end{array}\right] \\
& \mathbf{B}_{7}=\left[\begin{array}{cccccccccc} 
& 1 & 0 & 0 & 0 & 0 & 0 & 0 & 0 & \\
& 0 & 1 & 0 & 0 & 0 & 0 & 0 & 0 & \\
0 & 0 & 0 & 0 & 1 & 0 & 0 & 0 & 0 & 0 \\
(6 \times 20) & 0 & 0 & 0 & 0 & 0 & 1 & 0 & 0 & (6 \times 15) \\
& 0 & 0 & 0 & 0 & 0 & 0 & 1 & 0 & \\
& 0 & 0 & 0 & 0 & 0 & 0 & 0 & 1
\end{array}\right] \\
& \mathbf{B}_{8}=\left[\begin{array}{cccccc} 
& 0 & 0 & 0 & 0 & \\
& 0 & 0 & 0 & 0 & \\
0 & 1 & 0 & 0 & 0 & 0 \\
(6 \times 24) & 0 & 1 & 0 & 0 & (6 \times 15) \\
& 0 & 0 & 1 & 0 & \\
& 0 & 0 & 0 & 1
\end{array}\right]
\end{aligned}
$$

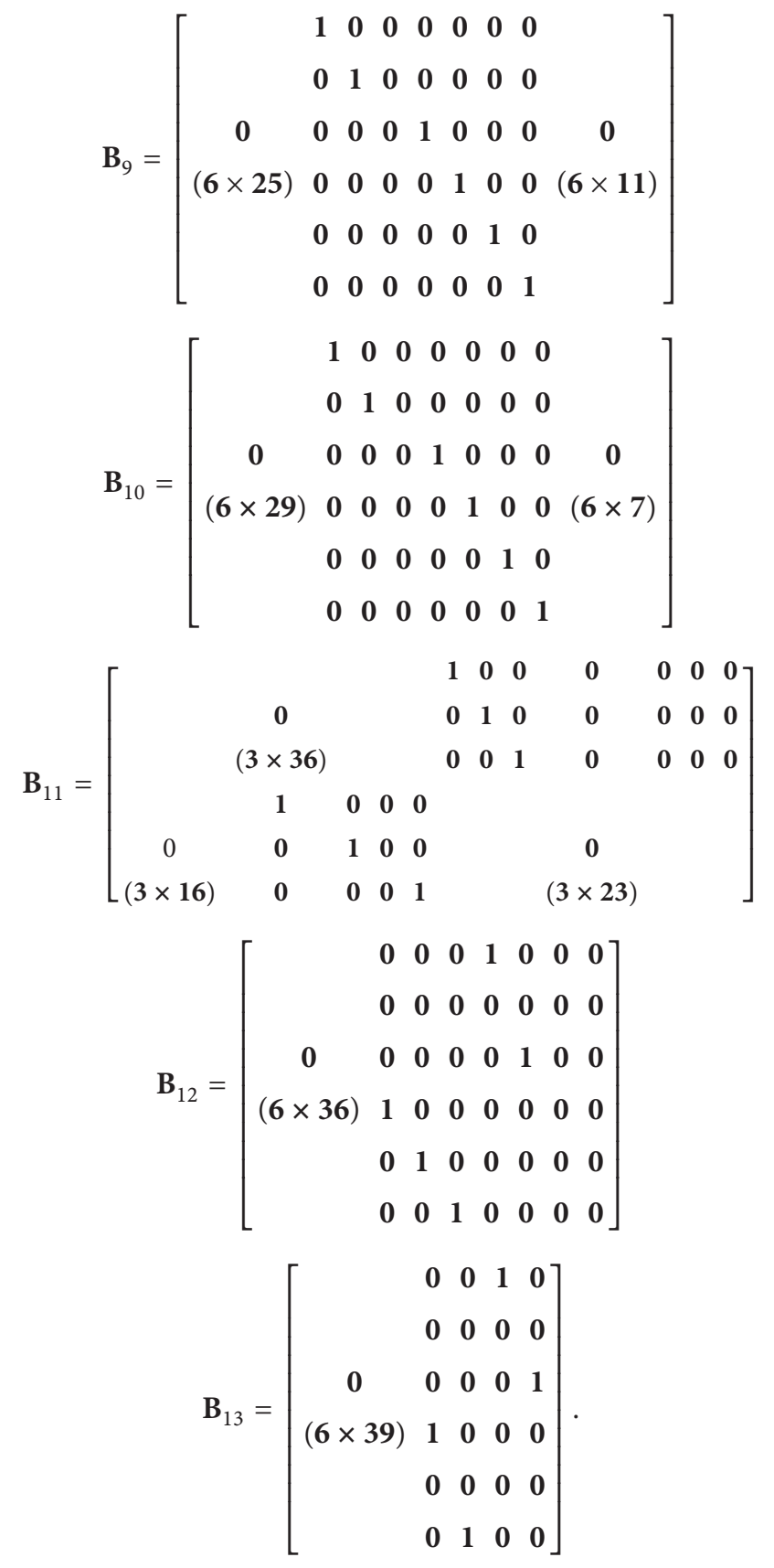

Similarly, there is the same relation between $\ddot{\mathbf{U}}_{r}$ and $\ddot{\mathbf{U}}_{r i}^{e}$, which can be denoted by formula

$$
\ddot{\mathbf{U}}_{r i}^{e}=\mathbf{B}_{i} \ddot{\mathbf{U}}_{r} \text {. }
$$

By substituting formula (29) into formula (22) and multiplying "on the left" on both sides, the following formula can be obtained:

$$
M_{i}^{e} \ddot{U}+K_{i}^{e} U=F_{i}^{e}-M_{i}^{e} \ddot{U}_{r}
$$

where

$$
\begin{array}{r}
M_{i}^{e}=B_{i}^{T} m_{i} B_{i} \quad K_{i}^{e}=B_{i}^{T} k_{i} B_{i} \\
(i=1,2, \ldots 13) .
\end{array}
$$




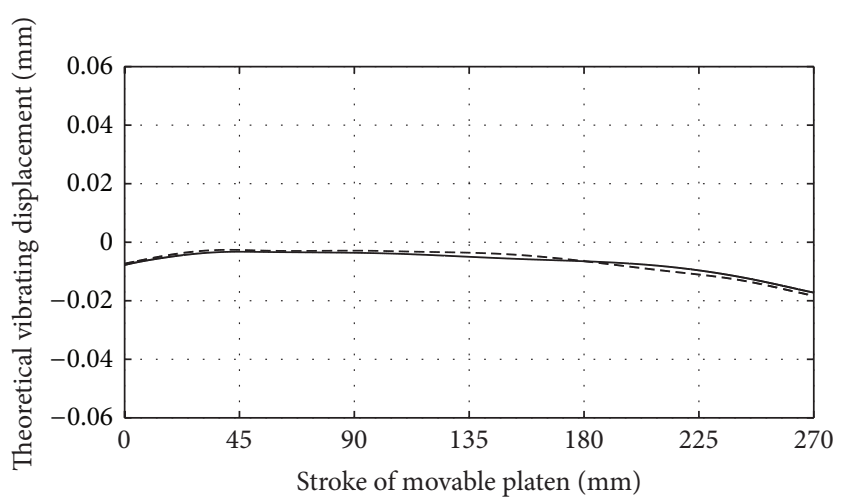

(a) Clamping speed $=100 \mathrm{~mm} / \mathrm{s}$

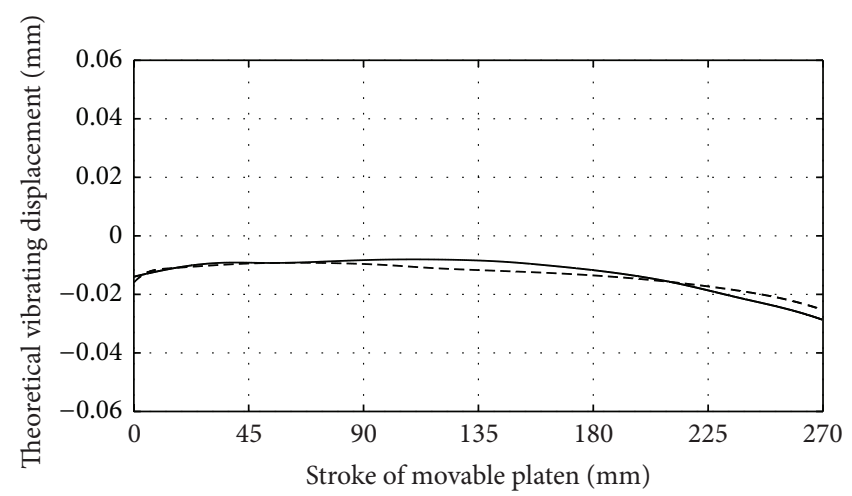

(b) Clamping speed $=300 \mathrm{~mm} / \mathrm{s}$

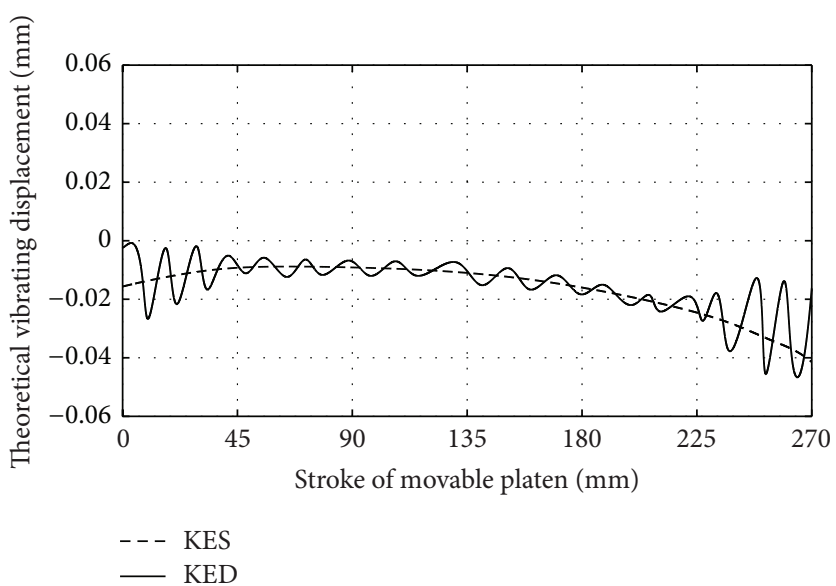

(c) Clamping speed $=500 \mathrm{~mm} / \mathrm{s}$

Figure 8: Vibrating displacement of $\mathbf{D}$ point in $X$ direction at different clamping speed.

Formula (30) is the equation of motion of the elements, in which the generalized coordinates with global numbers are considered as unknown quantities. By assembling the 13 equations of motion of the 13 elements, the differential equation of motion of the system can be obtained as formula

$$
\mathbf{M U}+\mathbf{K U}=\mathbf{F}-\ddot{M U}_{\mathbf{r}} .
$$

\section{KED and KES Simulation Analysis}

The key of elastic dynamic analysis for the clamping mechanism is to solve the differential equation of motion of the system. Formula (32) is identical in form with the differential equation for vibration of multi-degree-of-freedom systems. In the instantaneous kinematic analysis process, the mechanism is supposed to be frozen in some special orientation, while, in the actual working process, the orientation of the mechanism is changing, and the angle between the axis of the beam element and the $x$-axis of the fixed coordinate system varies with the orientation of the mechanism. Accordingly, the system mass matrix $\mathbf{M}$ and rigid matrix $\mathbf{K}$, which is composed of the element' mass matrix $\mathbf{m}$ and rigid matrix $\mathbf{k}$, are functions of the orientation of the mechanism. Therefore, the elastic dynamic equation (32) is a differential equation with variable coefficients, which could usually be solved by modal analysis method.

In this paper, we mainly focus on the establishment of the dynamic mode of the clamping mechanism but not on the solution of the dynamic mode, which will be explored in another paper.

Here, the complicated solving process is neglected, and the results by the method of Kineto-Elasto Dynamics (KED) and Kineto-Elasto Static (KES) are given directly. The comparison and analysis between the KED and KES results were done.

KED analysis is used to study the vibration of the clamping mechanism, which is considered as an elastic motion system and bears inertial forces and external loads, and then to calculate the displacement, velocity, acceleration, strain, and stress of each part in the mechanism. The fundamental difference between KES and KED is that, in KES analysis, the inertial forces and external loads are supposed as static loads.

In this study, a simulation case at different clamping speed was given, and the results from KED analysis and KES analysis are compared. Dimensions of the 13 beam elements for the computation of the clamping unit are shown in Table 1.

The following figures (from Figures 8-11) are the KED and KES analysis results at some special points of rod CD and rod 


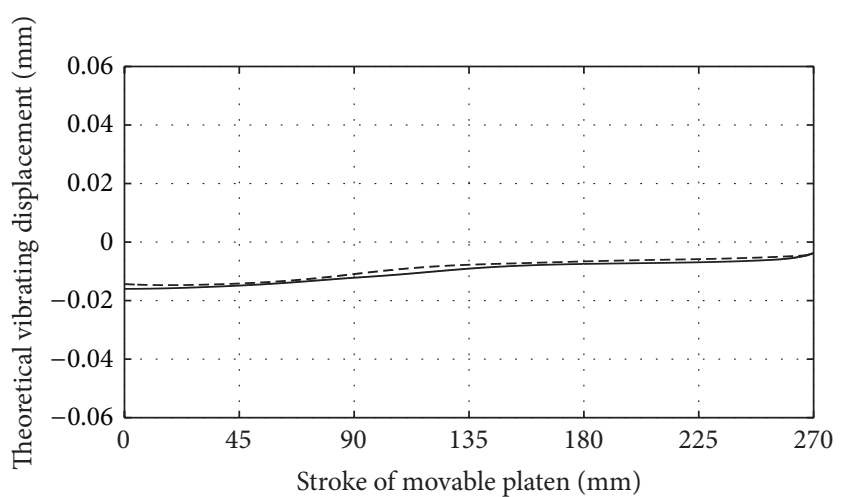

(a) Clamping speed $=100 \mathrm{~mm} / \mathrm{s}$

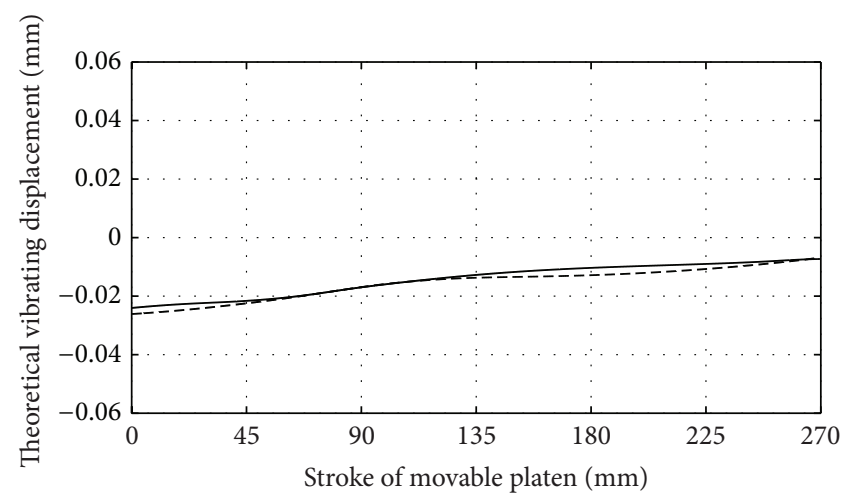

(b) Clamping speed $=300 \mathrm{~mm} / \mathrm{s}$

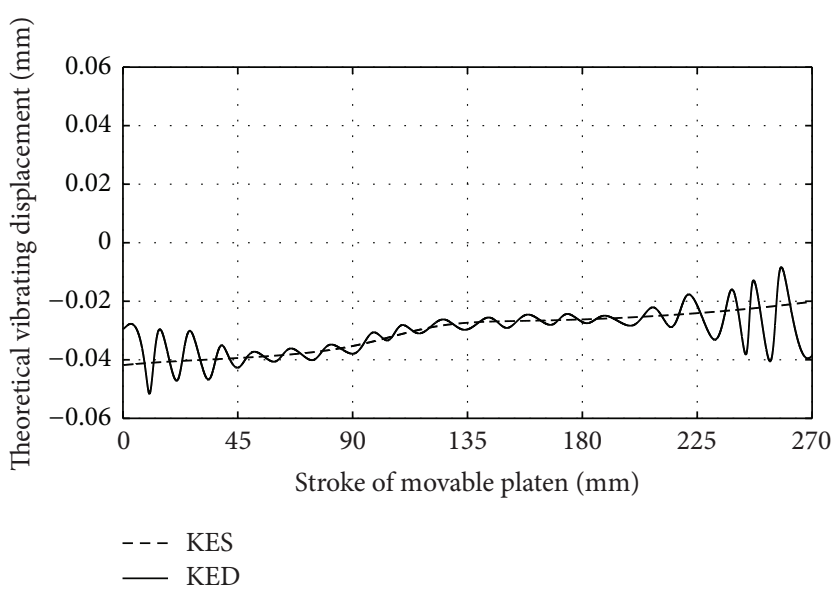

(c) Clamping speed $=500 \mathrm{~mm} / \mathrm{s}$

FIGURE 9: Vibrating displacement of $\mathbf{D}$ point in $Y$ direction at different clamping speed.

TABLE 1: Dimensions of beam elements for the computation case.

\begin{tabular}{lcc}
\hline Elements number & Length & Sectional dimension \\
\hline Element (1) & $168 \mathrm{~mm}$ & $50 \times 20 \mathrm{~mm}$ rectangle \\
Element (2) & $50 \mathrm{~mm}$ & $45 \times 20 \mathrm{~mm}$ rectangle \\
Element (3) & $227.5 \mathrm{~mm}$ & $50 \times 20 \mathrm{~mm}$ rectangle \\
Element (4) & $101.5 \mathrm{~mm}$ & $45 \times 20 \mathrm{~mm}$ rectangle \\
Element (5) & $115 \mathrm{~mm}$ & $50 \times 20 \mathrm{~mm}$ rectangle \\
Element (6) & $115 \mathrm{~mm}$ & $50 \times 20 \mathrm{~mm}$ rectangle \\
Element (7) & $101.5 \mathrm{~mm}$ & $45 \times 20 \mathrm{~mm}$ rectangle \\
Element (8) & $168 \mathrm{~mm}$ & $50 \times 20 \mathrm{~mm}$ rectangle \\
Element (9) & $50 \mathrm{~mm}$ & $45 \times 20 \mathrm{~mm}$ rectangle \\
Element (10) & $227.5 \mathrm{~mm}$ & $50 \times 20 \mathrm{~mm}$ rectangle \\
Element (11) & $98 \mathrm{~mm}$ & $14 \mathrm{~mm}$ radius circle \\
Element (12) & $120 \mathrm{~mm}$ & $32 \mathrm{~mm}$ radius circle \\
Element (13) & $360 \mathrm{~mm}$ & $32 \mathrm{~mm}$ radius circle \\
\hline
\end{tabular}

BK, where the abscissa is the stroke of the movable platen and the ordinate is the vibrating displacement of the special point at rod CD and rod BK. The black curves denote the results of KED analysis, and the red curves denote the ones of KES analysis.
Figures $8(\mathrm{a}), 8(\mathrm{~b})$, and $8(\mathrm{c})$ are the theoretical vibrating displacement curves of $\mathbf{D}$ point in $X$ direction, at 100, 300, and $500 \mathrm{~mm} / \mathrm{s}$ clamping speed, respectively. Figures $9(\mathrm{a})$, 9(b), and $9(\mathrm{c})$ are the ones of $\mathbf{D}$ point in $Y$ direction.

Figures $10(\mathrm{a}), 10(\mathrm{~b})$, and 10 (c) are the vibrating theoretical displacement curves of $\mathbf{K}$ point in $X$ direction, at 100, 300, and $500 \mathrm{~mm} / \mathrm{s}$ clamping speed, respectively. Figures $11(\mathrm{a})$, $11(\mathrm{~b})$, and $11(\mathrm{c})$ are the ones of $\mathbf{K}$ point in $Y$ direction.

The following can be seen from these figures.

(1) When the mechanism is clamping at the speed of $100 \mathrm{~mm} / \mathrm{s}$, the vibrating displacements of both $\mathbf{D}$ and $\mathbf{K}$ points in $X$ and $Y$ direction are very small in value, and the KED curve and KES curve almost coincide.

(2) When the mechanism is clamping at the speed of $300 \mathrm{~mm} / \mathrm{s}$, the KED curve and KES curve are similar, despite existing a litter difference, which indicates that it is feasible to use KES instead of KED to analyze the clamping process at low clamping speed.

(3) While at the clamping speed of $500 \mathrm{~mm} / \mathrm{s}$, there is an obvious and essential difference between the KES curve and KED curve. Namely, the KES curve is smooth, but the KED curve is fluctuant. At the beginning and end of the clamping process, the KED 


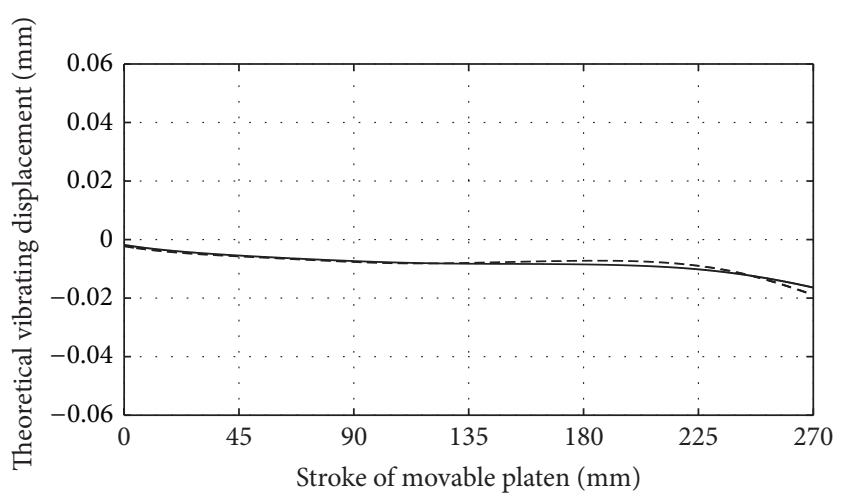

(a) Clamping speed $=100 \mathrm{~mm} / \mathrm{s}$

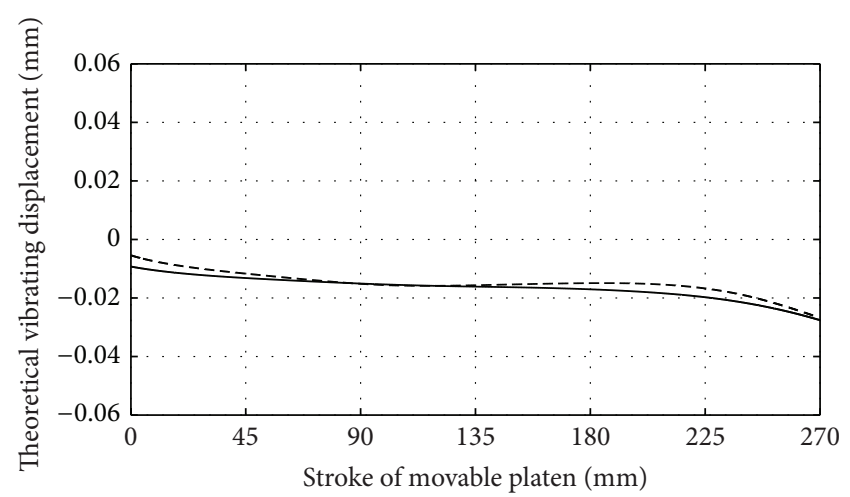

(b) Clamping speed $=300 \mathrm{~mm} / \mathrm{s}$

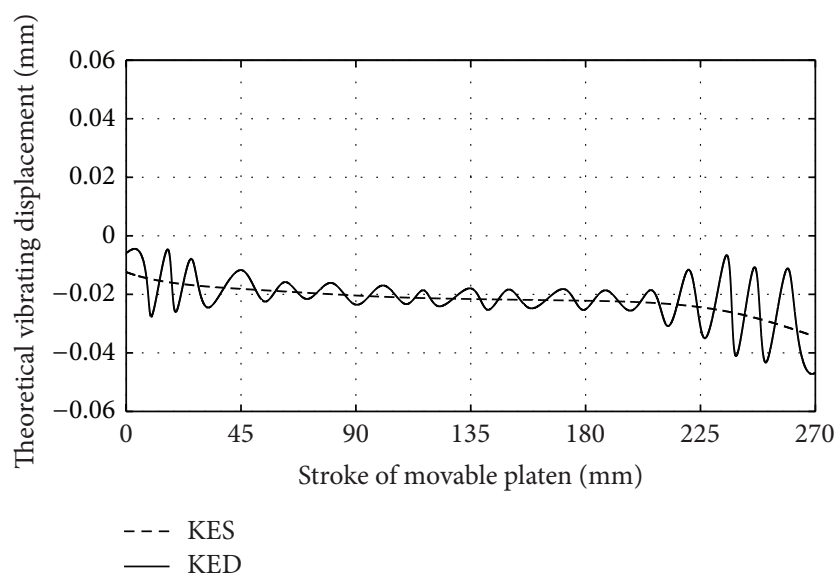

(c) Clamping speed $=500 \mathrm{~mm} / \mathrm{s}$

FIgURE 10: Vibrating displacement of $\mathbf{K}$ point in $X$ direction at different clamping speed.

curve changes suddenly with a larger vibrating displacement, due to the larger elastic deformation of the mechanism.

It is possible for the elastic deformation of the toggle rod to make the movable platen vibrating. The lateral elastic vibration of movable platen may intensify the friction and wear between the tie rods and the axle sleeves, while the longitudinal elastic vibration of movable platen may increase the clamping position error, cause impact on movable platen and stationary platen, and even damage the mould. Therefore, the traditional research method, which considers rods of the clamping mechanism as rigid bodies or quasi rigid bodies, is unsuited for the clamping system with high clamping speed. This will provide theoretical supports for the optimal design of clamping mechanism with high clamping speed, reducing the elastic vibration and improving the accuracy and steadiness of the clamping process.

As can be seen from Figures 8 to 11(c), at high clamping speed, the KED curves are well coincided with the KES curves in variation trend, despite the essential difference in the amplitude of vibrating displacements. The KES curves are very smooth without the effects of inertial forces and damping forces, and the KED curves fluctuate with the KES curves as center lines, which show that it is feasible to substitute KES for KED to study the vibration of the clamping mechanism at low clamping speed.

The elastic dynamic analysis was done to make all the parts of the clamping mechanism work elastically with uniform deformation amounts. The benefit is obvious. According to the solving results of the theoretical model, the elastic deformations of each part of the clamping mechanism are in an order of magnitude, which is especially important for the mechanism at high clamping speed. It is because that the enormous inertial forces, due to high working speed, may make the parts bear unbalanced forces and cause stress concentration, if the difference of strength, rigidity, and elasticity between parts is too large. The unbalanced forces and stress concentration are the major sources that cause damage to the plastic injection machine.

\section{Conclusions}

In this paper, based on appropriate assumptions and Lagrange equation, a high nonlinear and strong time-variant elastic dynamic model of the double-toggle clamping mechanisms was established. A simulation study on the elastic deformation and vibration of the clamping process was carried out by using the KED and KES analysis methods, respectively. The simulation results show that, at low 


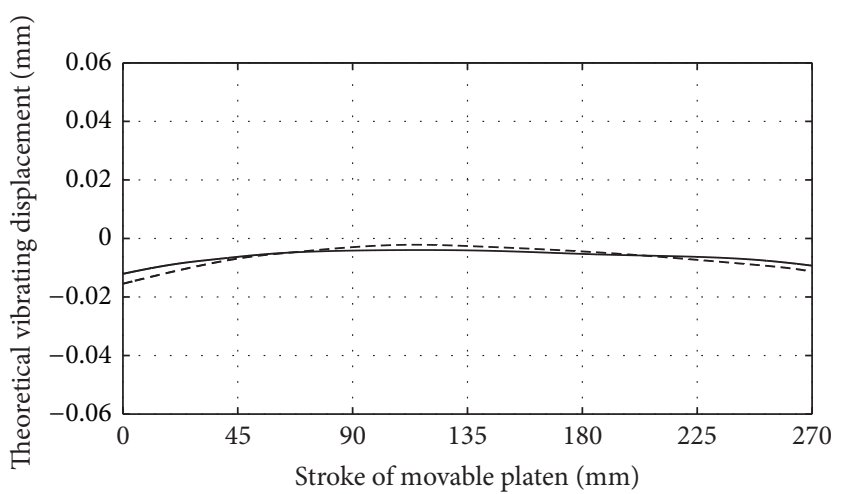

(a) Clamping speed $=100 \mathrm{~mm} / \mathrm{s}$

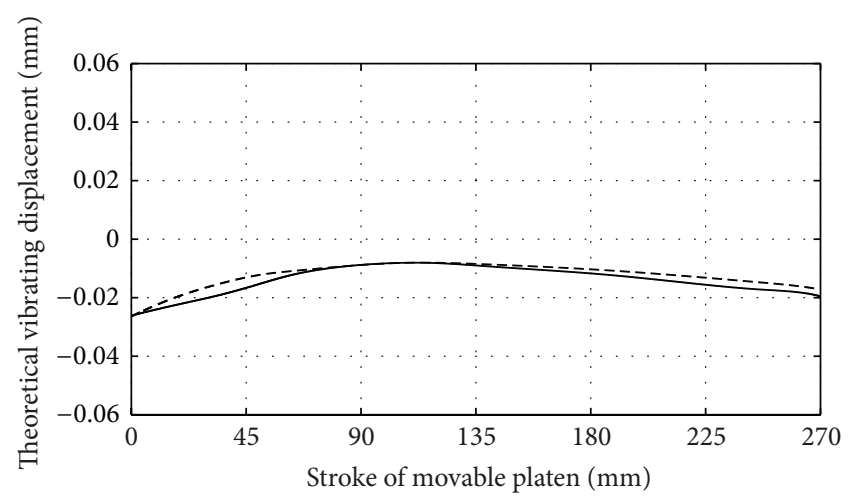

(b) Clamping speed $=300 \mathrm{~mm} / \mathrm{s}$

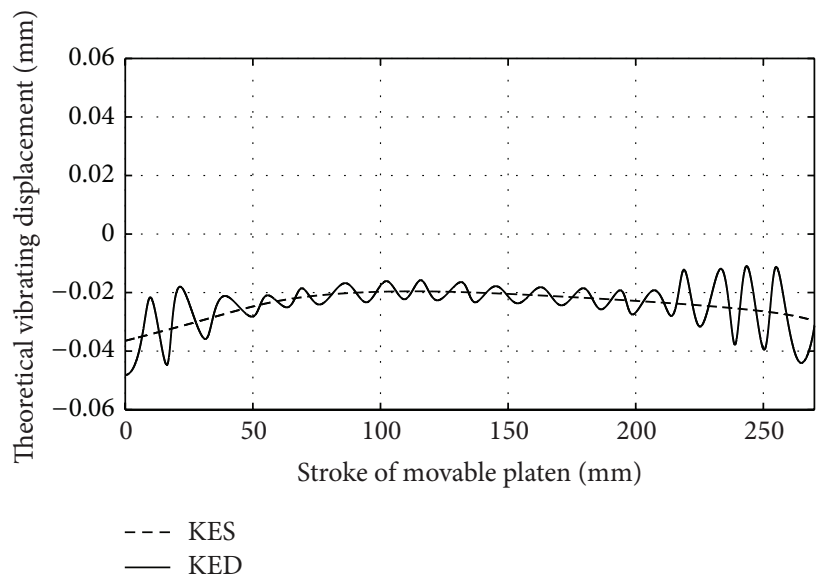

(c) Clamping speed $=500 \mathrm{~mm} / \mathrm{s}$

Figure 11: Vibrating displacement of $\mathbf{K}$ point in $Y$ direction at different clamping speed.

clamping speed, the elastic deformations of the toggle rods are very small, and the solving results of the two analysis methods coincide. While when the clamping speed is up to $500 \mathrm{~mm} / \mathrm{s}$, the toggle rods cause vibration and the KED curve changes suddenly with a larger vibrating displacement, at the beginning and at the end of the clamping process, due to the larger elastic deformation of the mechanism. This shows that the traditional research method, which considered rods of the clamping mechanism as rigid bodies, is unsuited for the clamping system with high clamping speed. This work will provide theoretical supports for the optimal design of clamping mechanism with high clamping speed, reducing the elastic vibration and improving the accuracy and steadiness of the clamping process.

\section{Nomenclature}

M: Mass matrix of system

C: Damp matrix of system

K: $\quad$ Rigid matrix of system

F: $\quad$ Generalized force matrix

$W$ : Displacement

$O X Y$ : Fixed coordinate system

$A \overline{X Y}$ : Moving coordinate system
Oxy: Rotary coordinate system

$U: \quad$ Generalized coordinate array

$\mathbf{U}^{e}: \quad$ Element generalized coordinate array

S: $\quad \sin \theta$

C: $\cos \theta$

$\dot{\mathbf{u}}_{a}$ : Absolute velocity array of element

$\dot{\mathbf{u}}_{r}$ : Rigid velocity array of element

u. $\quad$ Elastic velocity array of element

$\dot{\mathbf{u}}_{r e}$ : Relative velocity array of element

$\ddot{\mathbf{u}}_{n}$ : Relative normal acceleration array of element

$\ddot{\mathbf{u}}_{t}$ : $\quad$ Relative tangential acceleration array of element

$\ddot{\mathbf{u}}_{c}$ : Coriolis acceleration array of element

R: Coordinate transformation matrix

$\theta$ : Horizontal angle

$\ddot{\mathbf{U}}_{r}^{e}$ : Rigid acceleration array of element

$\mathbf{I}_{u}$ : $\quad$ Model matrix

$\dot{\mathbf{U}}_{r}$ : Rigid velocity array of system

$\ddot{\mathbf{U}}_{r}$ : Rigid acceleration array of system

$\mathbf{B}_{i}$ : Coordinate matrix

m: Mass matrix of element

c: Damp matrix of element

k: Rigid matrix of element. 


\section{Conflict of Interests}

The authors declare that there is no conflict of interests regarding the publication of this paper.

\section{Acknowledgments}

This work is supported by the Natural Science Foundation of Guangdong Province (S2012010010199) and Scientific and Technological Innovation Project of Foshan (2013AG100063), China.

\section{References}

[1] B. Ren, S. Zhang, and J. Tan, "Solution of rigid-flexible coupling problem in digital prototyping of double toggle mold closing device," Journal of Mechanical Engineering, vol. 48, no. 3, pp. 133-139, 2012.

[2] S.-W. Kim and L.-S. Turng, "Developments of three-dimensional computer-aided engineering simulation for injection moulding," Modelling and Simulation in Materials Science and Engineering, vol. 12, no. 3, pp. S151-S173, 2004.

[3] S. H. Sun, "Optimum topology design for the stationary platen of a plastic injection machine," Computers in Industry, vol. 55, no. 2, pp. 147-158, 2004.

[4] L. Quan and S. P. Liu, "Improve the kinetic performance of the pump controlled clamping unit in plastic injection molding machine with adaptive control strategy," Chinese Journal of Mechanical Engineering, vol. 19, no. 1, pp. 9-13, 2006.

[5] A. Pashkevich, A. Klimchik, and D. Chablat, "Stiffness analysis of parallel manipulators with preloaded passive joints," in Advances in Robot Kinematics: Motion in Man and Machine, pp. 465-474, Springer, 2010.

[6] A. Pashkevich, A. Klimchik, and D. Chablat, "Enhanced stiffness modeling of manipulators with passive joints," Mechanism and Machine Theory, vol. 46, no. 5, pp. 662-679, 2011.

[7] C. B. Drab, H. W. Engl, J. R. Haslinger, G. Offner, R. U. Pfau, and W. Zulehner, "Dynamic simulation of crankshaft multibody systems," Multibody System Dynamics, vol. 22, no. 2, pp. 133-144, 2009.

[8] Y. Yilmaz and G. Anlas, "An investigation of the effect of counterweight configuration on main bearing load and crankshaft bending stress," Advances in Engineering Software, vol. 40, no. 2, pp. 95-104, 2009.

[9] R. C. Winfrey, "Elastic link mechanism dynamics," ASME Journal of Engineering for Industry, vol. 93, no. 1, pp. 268-272, 1971.

[10] A. G. Erdman, G. N. Sandor, and R. G. Oakberg, "A general method for kineto elastodynamic analysis and synthesis of mechanisms," Journal of Engineering for Industry, vol. 94, no. 4, pp. 1193-1205, 1972.

[11] A. G. Erdman and G. N. Sandor, "Kineto-elastodynamicsa review of the state of the art and trends," Mechanism and Machine Theory, vol. 7, no. 1, pp. 19-33, 1972.

[12] C. Zhang, Machinery Dynamics, Higher Education Press, Beijing, China, 2000.

[13] H. M. Shi, Vibration Systems-Analyzing, Testing, Modeling, Controlling, Huazhong University of Science and Technology Press, Wuhan, China, 2nd edition, 2003. 

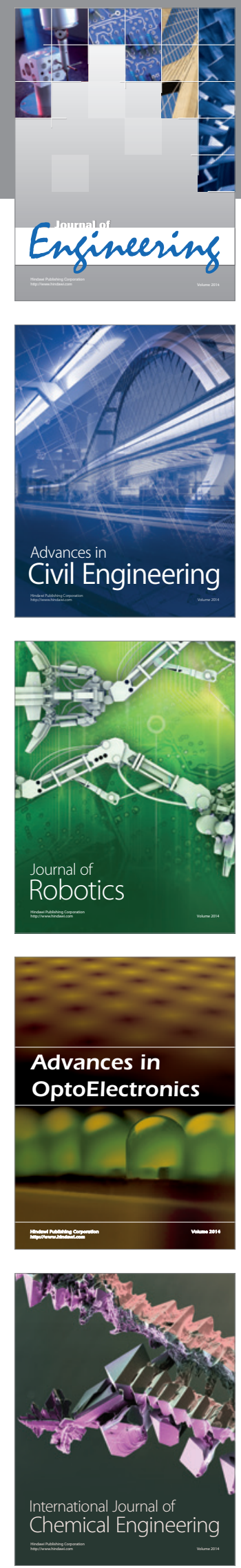

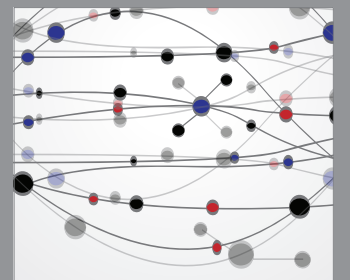

The Scientific World Journal
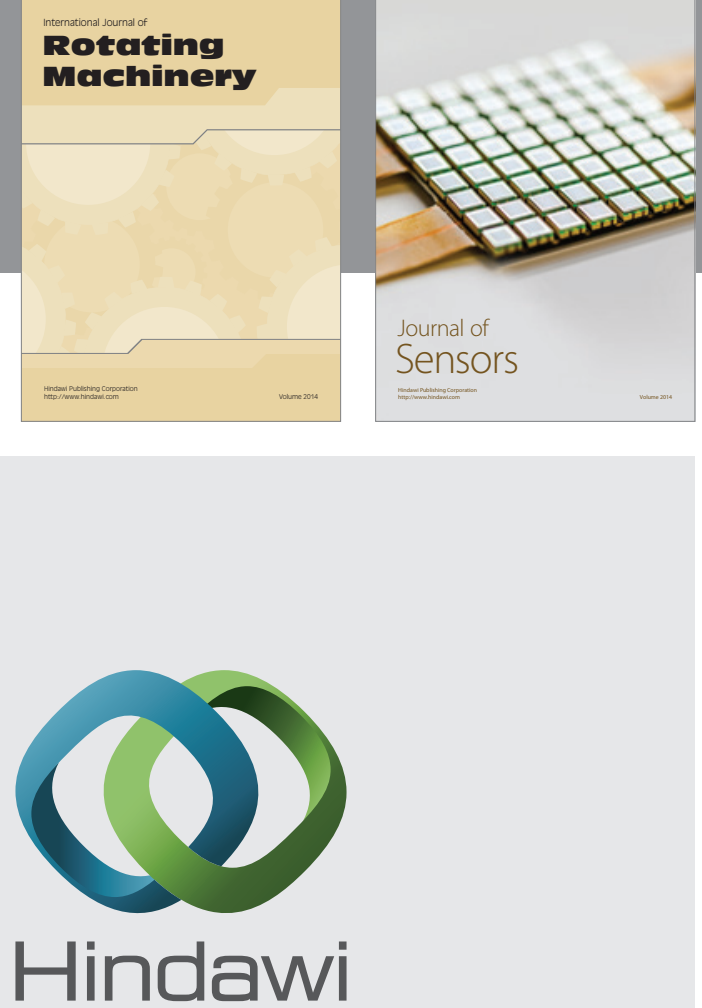

Submit your manuscripts at http://www.hindawi.com
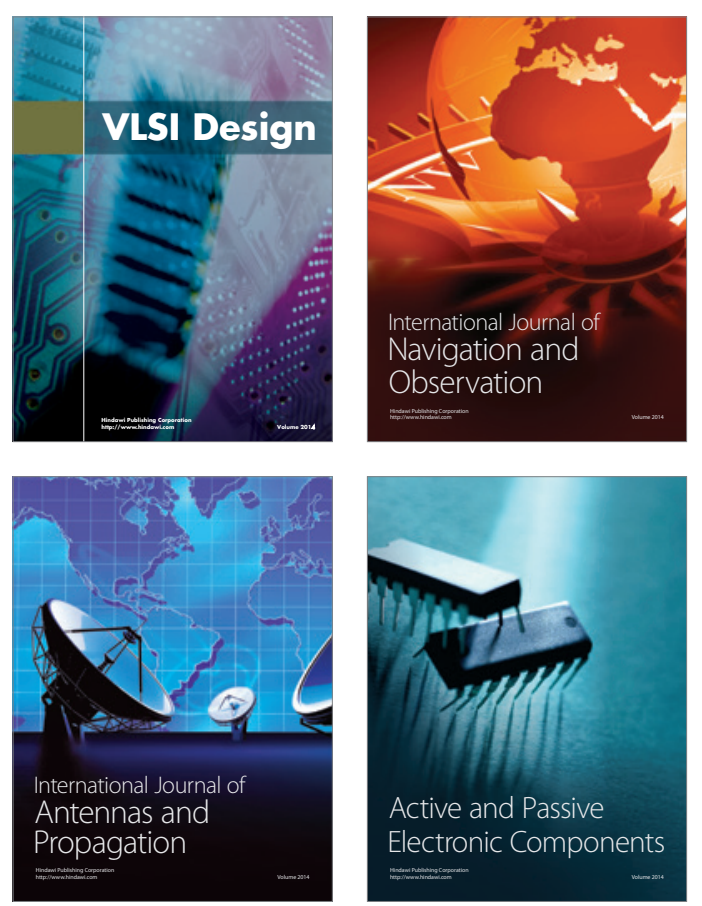
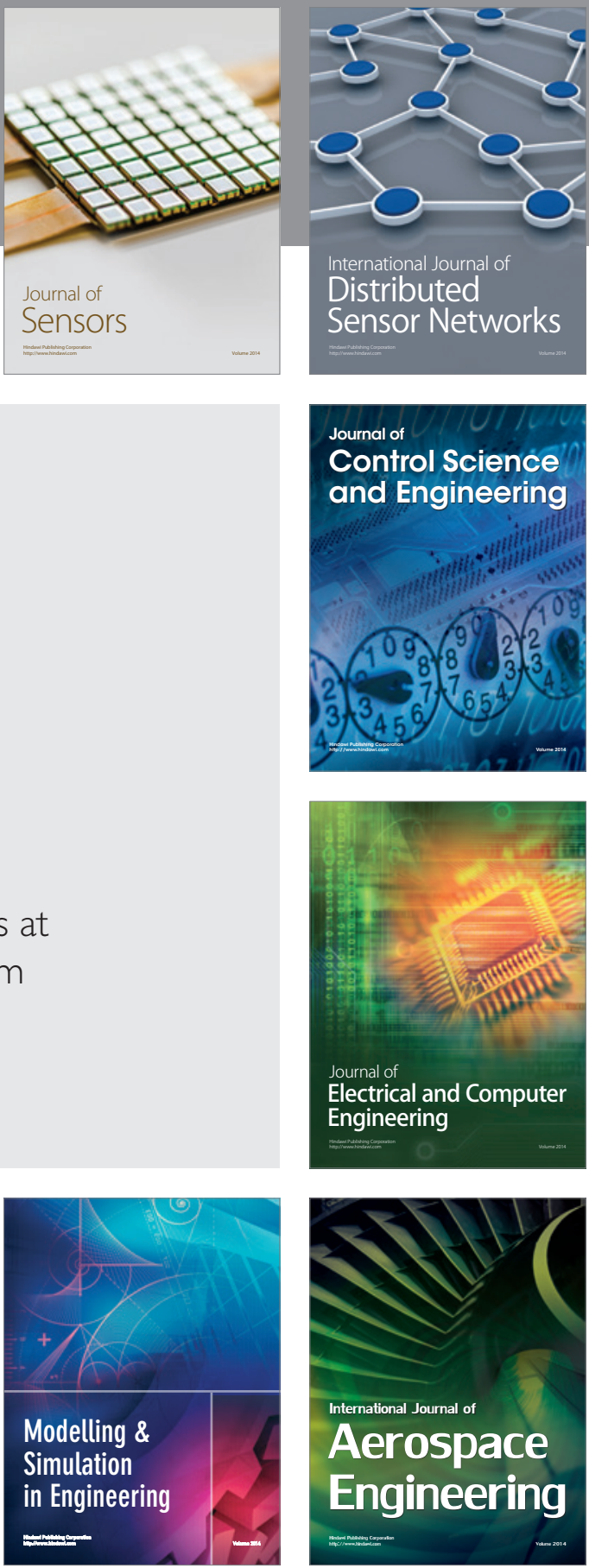

Journal of

Control Science

and Engineering
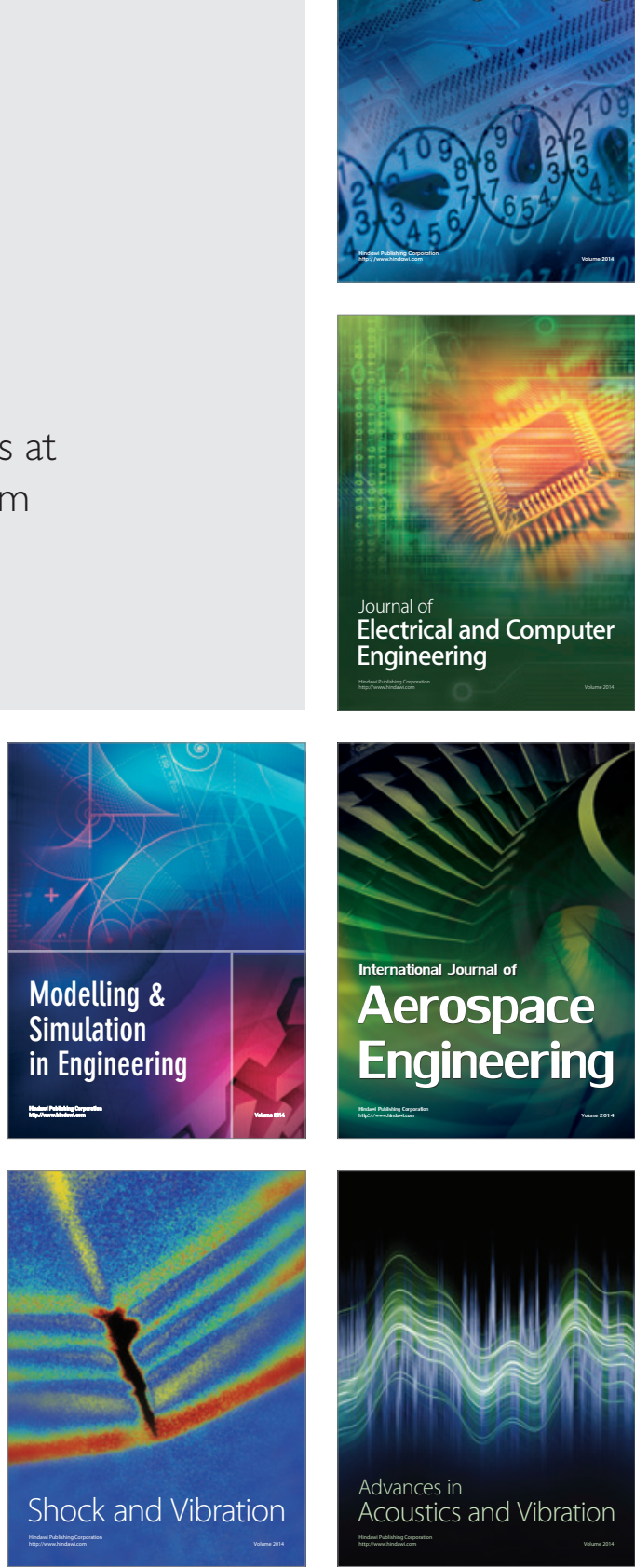